\title{
Síntesis de PIRIDIL-tolanos mediante un acoplamiento de sonogashira asistido por microondas
}

Synthesis of piridil-acetilene through a coupling of sonogashira assisted by microwaves

\author{
Linda Mariuxi Flores Fiallos. ${ }^{1}$ Cristina Nataly Villegas Freire. ${ }^{2} \&$ María Augusta \\ Guadalupe Alcoser. ${ }^{3}$
}

Recibido: 12-04-2020 / Revisado: 17-05-2020 / Aceptado: 20-06-2020 / Publicado: 03-07-2020

\begin{abstract}
.
DOI: https://doi.org/10.33262/cienciadigital.v4i3.1303

The use of the microwave in organic synthesis to obtain pyridyl tolans has been bet, since it is capable of providing enough energy in such a way that it is possible to cross a certain energy barrier, its use provides a decrease in the reaction time resulting in high performance products in which it was possible to limit the purification processes. Thus, in the present work, the synthesis of palladiumcatalyzed pyridyl-tolanes was carried out by means of a microwave-assisted Sonogashira coupling. The purification of the synthesized compounds was carried out by flash column chromatography using silica gel 60 GF254 with a positive pressure of nitrogen and with solvents suitable for the removal of undesirable inorganic matter ( $\mathrm{Cu}$ residues). The characterization was made using Nuclear Magnetic Resonance of both Hydrogen and Carbon, in order to demonstrate the presence of the triple bond formed with the Sonogashira coupling. Nuclear Magnetic Resonance Spectra were recorded on a Bruker AMX-300 spectrophotometer equipped with a $5 \mathrm{~mm}$ reverse multinuclear probe, using $\mathrm{CDCl} 3$ as the solvent. As a result, 2-pyridyl-tolane, 3-pyridyl-tolane and 4pyridyl-tolane were obtained, which were synthesized with acceptable yields of 71,73 and $78 \%$ respectively.
\end{abstract}

Keywords: Organometallic, coupling, Sonogashira, microwave.

\footnotetext{
${ }^{1}$ Escuela Superior Politécnica de Chimborazo, Facultad de Ciencias; Escuela de Ingeniería Química, Chimborazo - Ecuador, linda.flores@espoch.edu.ec

${ }^{2}$ Escuela Superior Politécnica de Chimborazo, Facultad de Recursos Naturales, Escuela de Ingeniería Forestal Chimborazo - Ecuador, cvillegas@espoch.edu.ec

${ }^{3}$ Escuela Superior Politécnica de Chimborazo, Proyecto registrado en el Instituto de Investigaciones de la ESPOCH Chimborazo - Ecuador, ma.augusta.guadalupe@gmail.com
} 


\section{Resumen.}

Se ha apostado al uso del microondas en la síntesis orgánica para la obtención de piridil tolanos, ya que es capaz de proporcionar suficiente energía de tal manera que es posible cruzar cierta barrera energética, su uso proporciona una disminución en el tiempo de reacción teniendo como resultado productos de rendimiento alto en los cuales fue posible limitar los procesos de purificación. Es así que en el presente trabajo se realizó la síntesis de piridil-tolanos catalizadas por paladio mediante un acoplamiento de Sonogashira asistido por microondas. La purificación de los compuestos sintetizados se realizó mediante cromatografía en columna ultrarrápida utilizando gel de sílice 60 GF254 con una presión positiva de nitrógeno y con disolventes adecuados para la remoción de materia inorgánica indeseable (residuos de $\mathrm{Cu}$ ). La caracterización se realizó mediante Resonancia Magnética Nuclear tanto de Hidrógeno como Carbono, para poder evidenciar así la presencia del triple enlace formado con el acoplamiento de Sonogashira. Se registraron s espectros de Resonancia Magnética Nuclear en un espectrofotómetro Bruker AMX-300 equipado con una sonda multinuclear inversa de $5 \mathrm{~mm}$, utilizando $\mathrm{CDCl} 3$ como disolvente. como resultado se obtuvieron el 2- piridiltolano, 3-piridil-tolano y el 4-piridil-tolano, que fueron sintetizados con rendimientos aceptables del 71,73 y $78 \%$ respectivamente.

Palabras clave: Organometálica, acoplamiento, Sonogashira, microondas.

\section{Introducción.}

El Debido a las propiedades de emisión de calor por parte del microondas se ha convertido en una alternativa valiosa en la síntesis de compuestos orgánicos, polímeros, materiales inorgánicos y nanomateriales (Kappe, Controlled Microwave Heating in Modern Organic Synthesis, 2004). Múltiples innovaciones importantes en el campo de la química asistida por microondas hoy en día permiten a los químicos preparar materiales catalíticos o nanomateriales y moléculas orgánicas deseadas, de forma selectiva, con rendimientos casi cuantitativos y con mayor precisión que utilizando el calentamiento convencional (Polshettiwar \& Varma, 2008). Al controlar los diversos parámetros específicos de microondas como temperatura, presión y rampa de temperatura, así como la correcta elección de los disolventes, los investigadores ahora pueden pasar a la próxima generación de diseño y desarrollo de nanomateriales avanzados (Kappe, Pieber, \& Dallinger, Microwave effects in organic synthesis: Myth or reality?, 2013).

En busca de solucionar los problemas que la industria enfrenta en síntesis convencional, múltiples investigadores trabajan en el diseño de nuevas técnicas, actualmente se han desarrollado síntesis de polímeros en microondas, usando tecnología supercrítica, o por extrusión reactiva, como alternativa a las técnicas convencionales.

La síntesis de polímeros que se encuentra en vía de desarrollo es la síntesis de polímeros asistida por microondas, que en un conjunto de trabajos realizados ha demostrado reducir 
considerablemente los tiempos de reacción, lo que supone costos energéticos más bajos, sistemas de reacción más seguros y mayor rendimiento en las reacciones (Garro, Jiménez, \& Vega, 2013). La fuente de calentamiento de microondas acelera las reacciones de policondensación (Mazo, Rios, \& Restrepo, 2011), incluso un estudio realizado, en el que se sintetiza polímeros tribloque demuestra que la técnica de irradiación de microondas fue más rápida que una síntesis en la cual se utilizó un disolvente supercrítico dióxido de carbono supercrítico (scCO2) (Kamali, Khodaverdi, \& Hadizadeh, 2018).

La síntesis asistida por microondas cumple las expectativas de ser una práctica de síntesis rápida. Desde los primeros informes en 1986, (Gedye, y otros, 1986) (Giguere, Bray, Duncan, \& Majetich, 1986), su uso ha sido importante en todo tipo de síntesis orgánica incluso en aquellas reacciones sin la presencia de disolvente o aquellas que emplean como disolvente agua (Gawande, Bonifacio, \& Luque, 2012). Aunque existe la controversia acerca de los "efectos no térmicos de emulación sobre el uso del microondas" (Dudley, Stiegman, \& Rosana, 2013), estudios realizados por Kappe ha demostrado que dichos efectos no existen (Kappe, Controlled Microwave Heating in Modern Organic Synthesis, 2004).

Las reacciones asistidas por microondas dependen tanto de la capacidad de la reacción para absorber energía y la elección del disolvente (alta absorción tan $\sigma>0.5$, medio 0.1 $<\tan <0.5$ y baja absorción tan $\sigma<0.1$ ) (Gabriel, Gabriel, Grant, Halstead, \& Mingos, 1998).

Actualmente el microondas es muy utilizado en reacciones de tipo organometálicas como es el caso de la reacción de Sonogashira, la cual data del año 1975 siendo un acoplamiento entre acetilenos terminales con haluros de arilo o vinilo para dar lugar alquil- y arilacetilenos así como eninos conjugados (Sonogashira, 2003). Se conoce que esta reacción fue estudiada de manera independiente por los grupos de Cassar (Cassar, 1975),Heck (Dick \& Heck, 1975) y Sonogashira (Sonogashira, 2003) .

Se conoce que dicha reacción necesita de la presencia de un co-catalizador de cobre ya que favorece al homoacoplamiento del alquino terminal (Sonogashira, 2003), en donde las condiciones de reacción varían dependiendo de los alquinos, haluros y bases utilizadas. El mecanismo llamado el ciclo del Paladio consiste en la formación de dos ciclos independientes en donde uno de ellos típicamente corresponde a la formación de enlaces C-C mediante una adición oxidante, transmetalación y oxidación reductora, en el segundo ciclo la amina juega un papel importante ya que es el precursor para la obtención del pre-catalizador de Pd(0) (Bertus, Fecourt, Bauder, \& Pale, 2004).

A parte del catalizador de paladio en la reacción de Sonogashira también debe estar presente ligantes a base de fósforo, con una pequeña cantidad catalítica de cobre $(\mathrm{Cu}(\mathrm{I}))$ y un exceso de una amina. Dentro de los ligantes fosfóricos más utilizados se encuentran el cloruro de bis (trifenilfosfina) paladio (II), 1,2-bis(difenilfosfina) etano (Hopkins \& Collar, 2004), debido a que por la riqueza electrónica presentes en estos ligantes favorece al paso limitante de la reacción es decir a la adición oxidativa. 
Mediante el acoplamiento de Sonogashira se han podido obtener: polímeros (Paresh, 2010), cristales líquidos (Nguyen, Horton, Hursthouse, Legon, \& Bruce, 2004), nuevos materiales supramoleculares con usos electrónicos (Gou, Chipara, \& Zaleski, 2007).

Con los antecedentes ya descritos el presente trabajo tiene como objetivo Sintetizar los N-Hetero-Tolanos mediante un acoplamiento de Sonogashira a partir del 1-etinil-4pentilfenilo y las respectivas yodo y bromo piridinas asistido por microondas, el método utilizado para la purificación fue la cromatografía en columna debido a su alta eficacia cuyo éxito radica en la elección adecuada de solventes para separar la fase móvil (materia inorgánica) de la fase estacionaria (materia orgánica), los productos fueron caracterizados por Resonancia Magnética Nuclear Carbono e Hidrógeno, técnica que permitió identificar la formación del triple enlace teniendo como resultado estructuras coloidales con características sólidas aportada por los aromáticos y líquida aportada por la cadena alquílica.

Es importante la elección de disolventes deuterados, para registrar el espectro de una muestra por RMN se prepara una disolución en un disolvente adecuado. Antes se usaba tetracloruro de carbono, puesto que no tiene átomos de hidrógeno que pudieran dar señal en RMN. Desafortunadamente el $\mathrm{CCl} 4$ es un mal disolvente para muchos compuestos polares y además es tóxico.

Actualmente se emplean disolventes marcados con deuterio, tales como chloroform-d (DCCl3), benzene-d6 (C6D6), acetone-d6 (CD3 COCD3) y DMSO-d6 (CD3SOCD3). Puesto que el deuterio tiene diferente momento magnético que el hidrógeno, no da señal cuando se registra un espectro de RMN ajustado al protón.

\section{Materiales y Métodos.}

Los compuestos utilizados para realizar la síntesis no tuvieron una previa purificación y fueron adquiridos de las siguientes casas comerciales Sigma Aldrich, Johnson Matthey, VWR.

La manipulación de todos los compuestos sensibles al aire y / o al agua se llevó a cabo usando técnicas estándar de atmósfera inerte (Bunge, y otros, 2003). Todos los productos químicos se usaron como se recibieron de fuentes comerciales sin más purificación. Se usaron solventes anhidros como se recibieron de fuentes comerciales. La cromatografía en capa fina analítica (TLC) se llevó a cabo en placas de gel de sílice con respaldo de aluminio Merck® 60 GF254 y la visualización, cuando se requirió, se logró usando luz UV o I2. La cromatografía en columna ultrarrápida se realizó en gel de sílice 60 GF254 usando una presión positiva de nitrógeno con el sistema disolvente indicado. Cuando se usaron mezclas de disolventes, las relaciones se informan por volumen. Los espectros de resonancia magnética nuclear se registraron en espectrómetros de $400 \mathrm{MHz}$ a temperatura ambiente de la sonda. Los desplazamientos químicos para los espectros de $1 \mathrm{H}$ RMN se registran en partes por millón a partir de tetrametilsilano con la resonancia del disolvente como patrón interno (cloroformo: $\delta=7,26 \mathrm{ppm}$ ). Los espectros de 13C NMR se 
registraron con desacoplamiento de protones completo. Los desplazamientos químicos se informan en partes por millón a partir de tetrametilsilano con la resonancia del disolvente como patrón interno (13CDCl3: 77.16 ppm).

Los espectros de Resonancia Magnética Nuclear fueron registrados en un espectrofotómetro Bruker AMX-300 equipado con una sonda multinuclear inversa de $5 \mathrm{~mm}$, utilizando $\mathrm{CDCl} 3$ como disolvente. Dicho equipo permite realizar experimentos con gradientes de campo magnéticos y detectar las señales de prácticamente todos los núcleos sensibles a la Resonancia Magnética Nuclear.

\section{Resultados y Discusión.}

Para la optimización de la reacción se examinó disolventes bases y condiciones para el calentamiento por microondas. El disolvente fue escogido según la capacidad que tuvo para convertir la energía del microondas en calor, el cual está determinado por la llamada pérdida de tangente $(\sigma)$, como se puede evidenciar en la tabla 1 (Paresh, 2010), por lo que se determinó que el mejor disolvente a utilizar fue el Tetrahidrofurano (THF) ya que se encuentra dentro de los mejores parámetros para ser utilizado en el microondas. El tiempo total de reacción para la síntesis de los productos deseados fue de 2 horas para cada uno, mientras que la misma síntesis mediante métodos convencionales resultó un tiempo total de tres días, con rendimientos inferiores al 30\% evidenciando un consumo mínimo de reactivo, y un mayor consumo de disolventes utilizados para separar la fase móvil de la fase estacionaria implicados en la purificación tanto de cromatografía en columna y cromatografía en capa fina.

En cuanto al catalizador se decidió aplicar 3.5\% en moles de paladio para todos los acoplamientos de Sonogashira para asegurar conversiones máximas y reacciones rápidas.

Se ha sintetizado y caracterizado, tres compuestos de Piridil Tolanos: 2-Piridil-Tolano, 3Piridil-Tolano y 4-Piridil-Tolano; mismos que fueron sintetizados mediante reacciones de acoplamiento de Sonogashira, acoplamiento del terminal fenílico (1-etinil-4pentilbenceno) con las yodo y bromo-piridinas tal como lo muestra el esquema 1 .

Tabla 1. Tangentes de pérdida de disolvente en reacciones asistidas por microondas

\begin{tabular}{cccc}
\hline SOLVENTE & $\tan \boldsymbol{\sigma}$ & SOLVENTE & $\tan \boldsymbol{\sigma}$ \\
\hline Etilenglicol & 1.350 & 1,2-dicloroetano & 0.127 \\
Etanol & 0.941 & Agua & 0.123 \\
$\begin{array}{c}\text { Dimetilsulfóxido } \\
\text { (DMSO) }\end{array}$ & 0.825 & Cloroformo & 0.091 \\
Metanol & 0.659 & Acetonitrilo & 0.062 \\
1,2-diclorobenceno & 0.280 & Tetrahidrofurano & 0.047
\end{tabular}




\begin{tabular}{cccc}
$N$-metil-2-pirrolidona & 0.275 & Diclorometano & 0.042 \\
Ácido acético & 0.174 & Tolueno & 0.040 \\
$\begin{array}{c}\text { Dimetilformamida } \\
(\mathrm{DMF})\end{array}$ & 0.161 & Hexano & 0.020 \\
\hline
\end{tabular}

Fuente: Elaboración propia.

Figura 1. Síntesis de N-Hetero-Tolanos.

a)

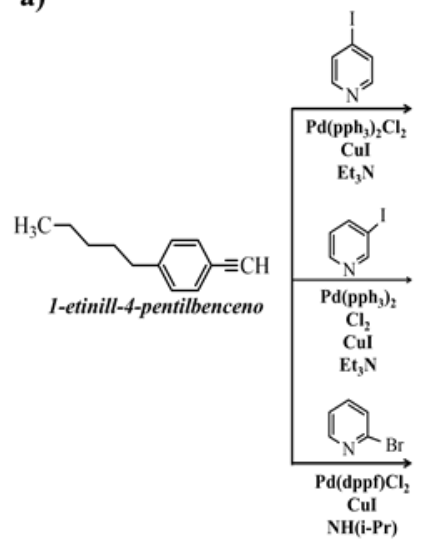

b)

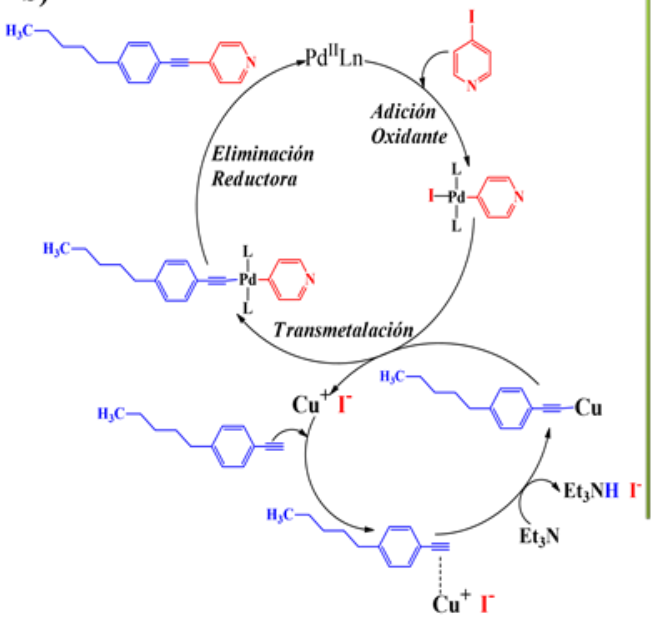

c)
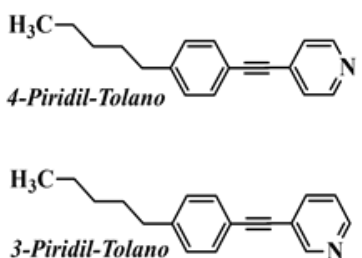

2-Piridil-Tolano

a) Condiciones de Rx; b) Acoplamiento de Sonogashira; c) Productos obtenidos.

Fuente: Elaboración propia.

Síntesis del 3 y 4-Piridil-Tolano: En un tubo Schlenk, se disolvió 1-etinil-4-pentilbenceno $(0,6 \mathrm{ml}, 3,1 \mathrm{mmol})$ en una mezcla de Tetrahidrofurano (THF) seco $(2 \mathrm{ml})$ y trietilamina (2 ml) y la solución se desgasificó mediante tres ciclos de congelación-descongelación antes de purgar con argón. Después se añadieron 4-yodopiridina o 3-yodopiridina (454 $\mathrm{mg}, 2,2 \mathrm{mmol}$ ), cloruro de bis (trifenilfosfina) paladio (II) $(78 \mathrm{mg}, 0,11 \mathrm{mmol}$ ) y yoduro de cobre (I) (42 mg, 0,22 mmol) a la solución, luego se colocó en el microondas durante 2 horas. La mezcla se diluyó a continuación con $\mathrm{CH} 2 \mathrm{Cl} 2(100 \mathrm{ml})$ y se lavó con agua $(100 \mathrm{ml}), \mathrm{NH} 4 \mathrm{Cl}$ acuoso saturado $(100 \mathrm{ml})$ y salmuera $(100 \mathrm{ml})$. La capa orgánica se secó sobre $\mathrm{MgSO} 4$ anhidro y los disolventes se eliminaron por evaporación rotatoria. El producto bruto fue purificado adicionalmente por cromatografía ultrarrápida ( $\mathrm{SiO} 2$, $\mathrm{CHCl} 3$ y luego $\mathrm{CHCl} 3$ con $1 \%$ de metanol).

En la figura 1 se indica la Resonancia Magnética Nuclear 1H-RMN (400 MHz, CDCl3) en el cual se evidencia los siguientes resultados:

El compuesto 4-Piridil-Tolano se aisló como un sólido marrón. Rendimiento: 374 mg (68\%). 1H NMR (400 MHz, CDCl3) $\delta H 8.58$ (dd, J = 4.5, 1.5 Hz, 2H), 7.47 (d, J = 8.1 
$\mathrm{Hz}, 2 \mathrm{H}), 7.36(\mathrm{dd}, \mathrm{J}=4.5,1.5 \mathrm{~Hz}, 2 \mathrm{H}), 7.19(\mathrm{~d}, \mathrm{~J}=8.1 \mathrm{~Hz}, 2 \mathrm{H}), 2.61(\mathrm{t}, \mathrm{J}=7.5 \mathrm{~Hz}, 2 \mathrm{H})$, 1.61 (quint, $\mathrm{J}=7.5 \mathrm{~Hz}, 2 \mathrm{H}), 1.31(\mathrm{~m}, 4 \mathrm{H}), 0.89$ (t, $\mathrm{J}=6.8 \mathrm{~Hz}, 3 \mathrm{H}$ ); (Figura 1)

13C NMR (100 MHz, CDCl3) $\delta \mathrm{C} 149.56,144.37,131.70,131.51,128.46,125.32$, $119.13,94.28,86.08,35.77,31.30,30.70,22.38$, 13.89. (Figura 2)

Figura 2. Resonancia Magnética Nuclear 1H-RMN (400 MHz, CDCl3)
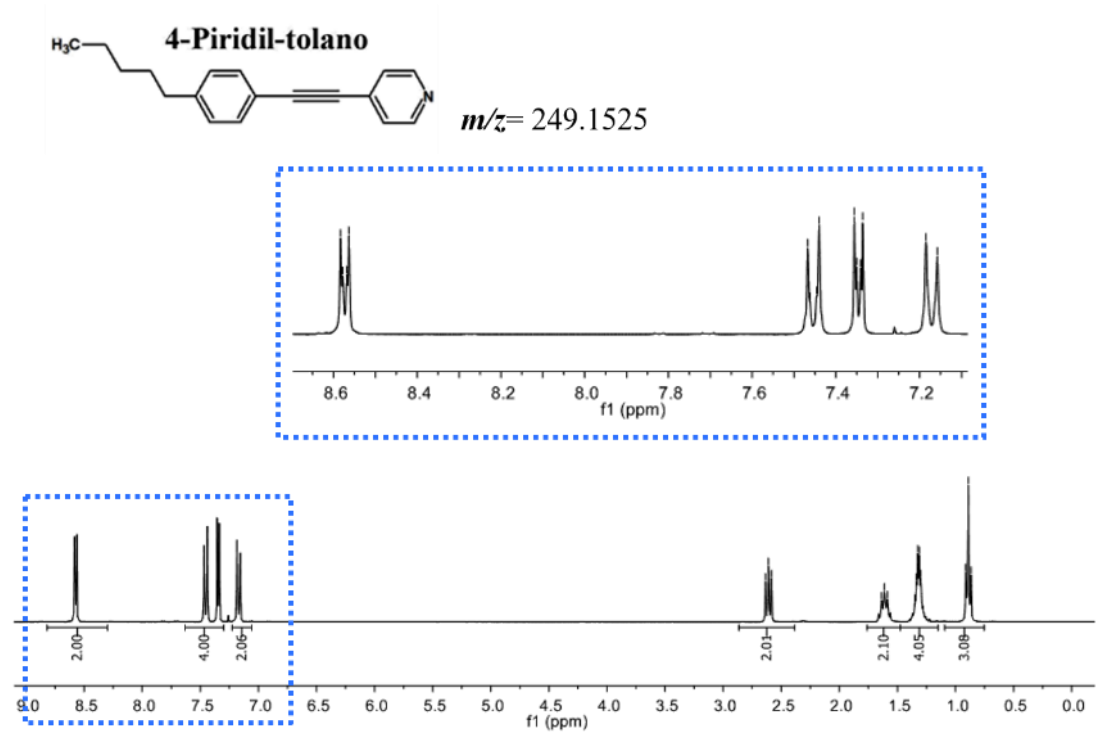

Fuente: Elaboración propia.

Figura 3. Resonancia Magnética Nuclear 13C (400 MHz, CDCl3)
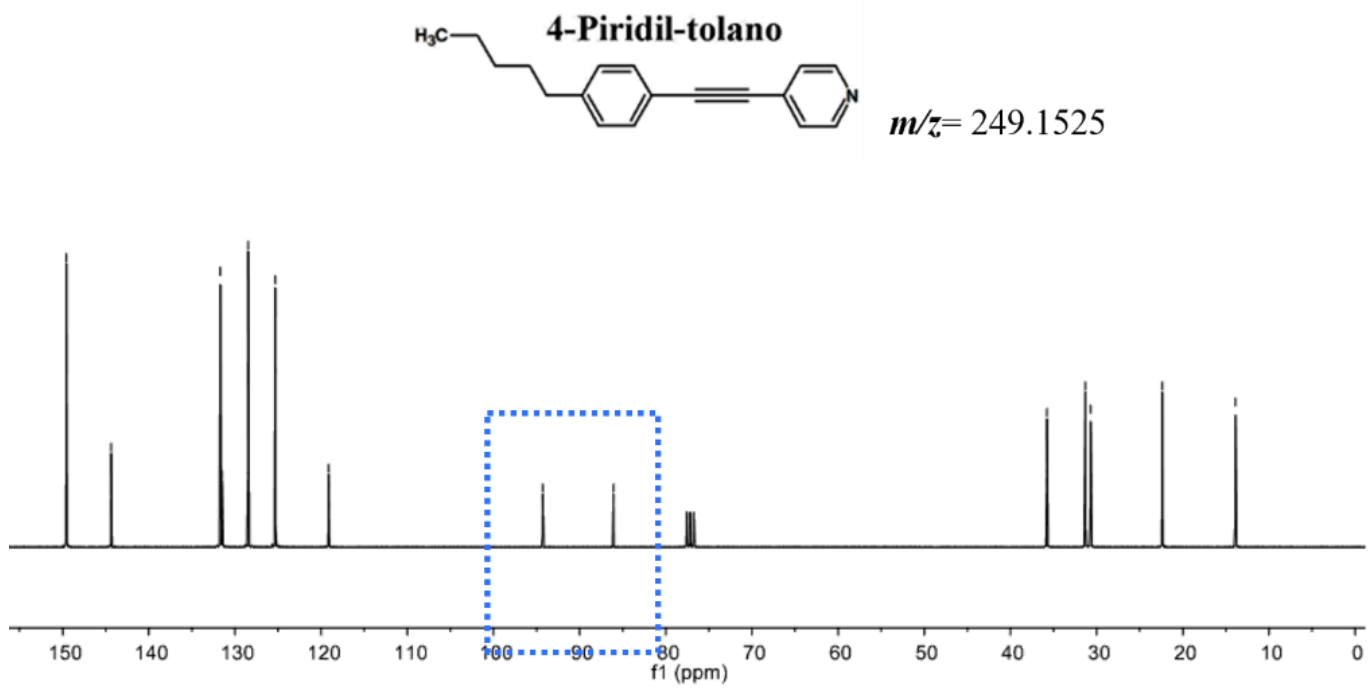

Fuente: Elaboración propia.

El compuesto 3-Piridil-Tolano se aisló como un aceite marrón. Rendimiento: $402 \mathrm{mg}$ (73\%). 1H NMR (400 MHz, CDCl3) $\delta \mathrm{H} 8.76$ (dd, J = 1.3,1H), $8.54(\mathrm{dd}, \mathrm{J}=4.9,1.7 \mathrm{~Hz}$, $1 \mathrm{H}), 7.81(\mathrm{dt}, \mathrm{J}=7.9,1.7,1.3 \mathrm{~Hz}, 1 \mathrm{H}), 7.47(\mathrm{~d}, \mathrm{~J}=8.3 \mathrm{~Hz}, 2 \mathrm{H}), 7.29(\mathrm{td}, \mathrm{J}=7.9,4.9,0.8$ 
$\mathrm{Hz}, 1 \mathrm{H}), 7.19$ (d, J = 8.3 Hz, 2H), 2.62 (t, J = 7.3 Hz, 2H), 1.62 (quint, J = 7.3 Hz, 2H), $1.31(\mathrm{~m}, 4 \mathrm{H}), 0.89$ (t, J = 6.9 Hz, 3H). (Figura 3)

13C NMR (100 MHz, CDCl3) \&C 152.13, 148.27, 143.94, 138.17, 131.55, 128.48, $122.87,120.62,119.62,92.93,85.33,35.81,31.36,30.79,22.45,13.95$. (Figura 4)

Figura 4. Resonancia Magnética Nuclear 1H-RMN (400 MHz, CDCl3)

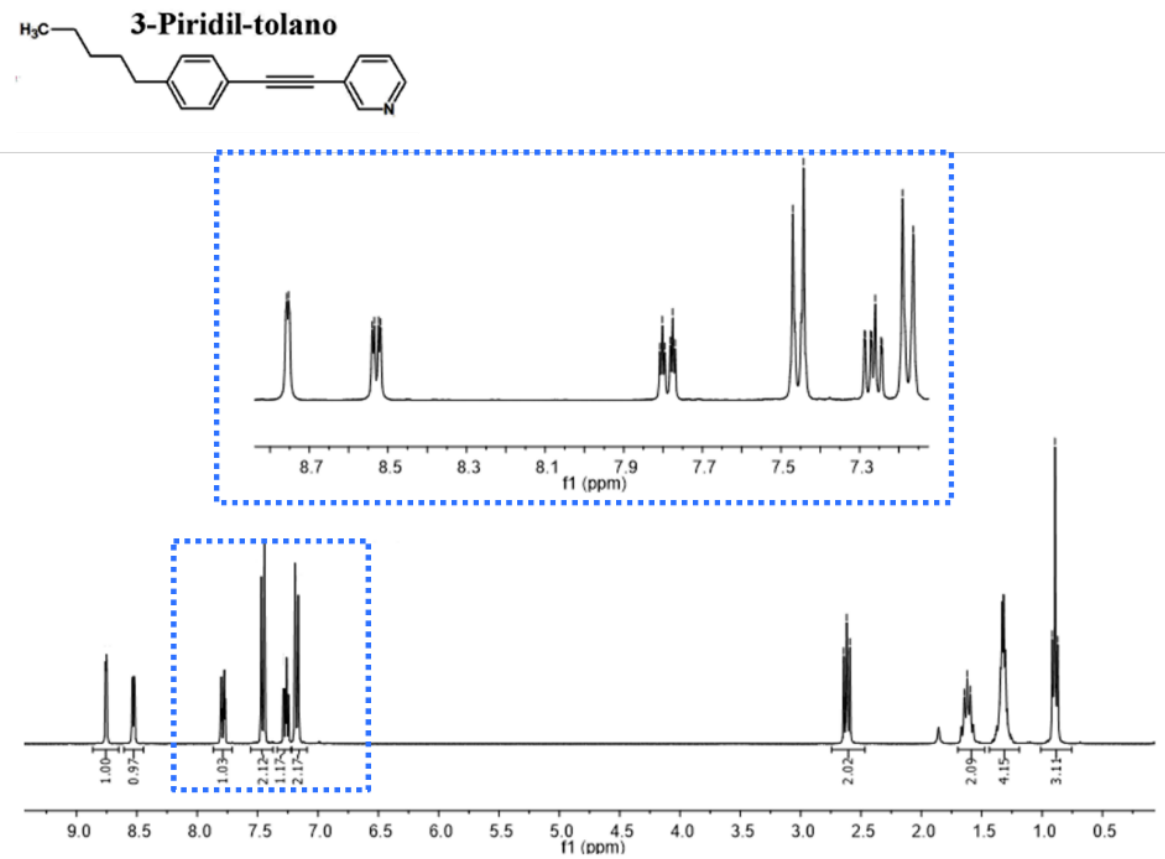

Fuente: Elaboración propia.

Figura 5. Resonancia Magnética Nuclear 13C (400 MHz, CDCl3)

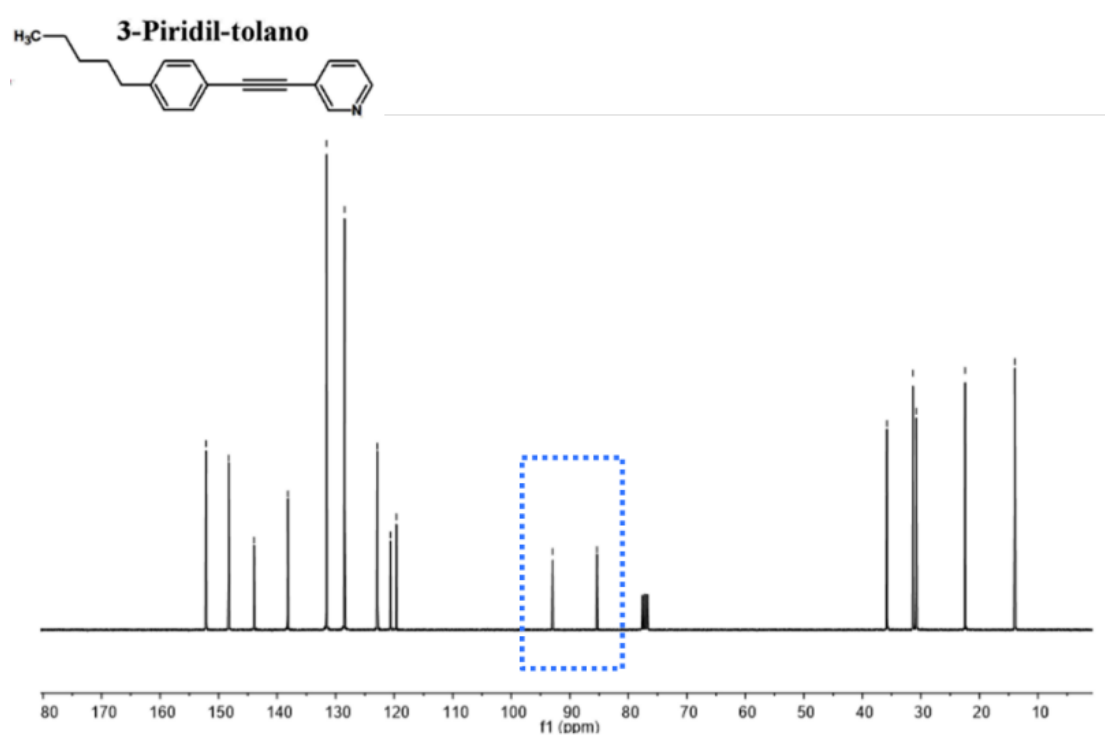

Fuente: Elaboración propia. 
Síntesis 2-Piridil-Tolano: En un reactor pequeño para microondas se colocó 1-etinil-4pentilbenceno ( $50 \mu 1,0.949 \mathrm{mmol})$ conjuntamente con 2-bromopiridina (230 $\mu 1,1.139$ $\mathrm{mmol})$ en una mezcla de THF seco $(2 \mathrm{ml})$ y trietilamina $(2 \mathrm{ml})$. Después se añadió bis (difenilfosfina) ferroceno de cloro paladio (II) $(33.8 \mathrm{mg}, 0,04 \mathrm{mmol}$ ) y yoduro de cobre (I) (9.1 mg, 0,22 mmol) a la solución. La reacción se llevó a cabo en microondas en un tiempo de 2 horas, una vez que se enfrió la disolución se procedió a recristalizarlo con Hexano caliente en donde se observó la separación de dos fases una sólida y una líquida, se llevó a centrifugación por el transcurso de 20 min, cuidadosamente se separó la parte sólida (de color negro intenso-impurezas) de la líquida (de color amarillo).La mezcla se diluyó a continuación con $\mathrm{CH} 2 \mathrm{Cl} 2(50 \mathrm{ml})$ y se lavó con agua $(50 \mathrm{ml}), \mathrm{NH} 4 \mathrm{Cl}$ acuoso saturado $(50 \mathrm{ml})$ realizada una cromatografia en capa fina TLC se vio necesario realizar cromatografía por columna ( $\mathrm{CHCl} 2$ con $1 \%$ de metanol).

El compuesto 2-Piridil-Tolano se aisló como un aceite amarillo. Rendimiento: $232 \mathrm{mg}$ (71\%) 1H NMR (400 MHz, CDCl3) $\delta 8.62$ (d, J = 4.5 Hz, 1H), 7.68 (td, J = 7.7, 1.8 Hz, $1 \mathrm{H}), 7.51(\mathrm{~d}, \mathrm{~J}=8.2 \mathrm{~Hz}, 2 \mathrm{H}), 7.50(\mathrm{~d}, \mathrm{~J}=7.7 \mathrm{Hx}), 7.23(\mathrm{~m}, \mathrm{~J}=7.7,2.8,1.8 \mathrm{~Hz}, 1 \mathrm{H}), 7.17$ $(\mathrm{d}, \mathrm{J}=8.0 \mathrm{~Hz}, 2 \mathrm{H}), 2.72-2.51(\mathrm{~m}, 2 \mathrm{H}), 1.65-1.57(\mathrm{~m}, 2 \mathrm{H}), 1.38-1.27(\mathrm{~m}, 4 \mathrm{H}), 0.97$ $-0.82(\mathrm{~m}, 3 \mathrm{H})$. (Figura 5)

13C NMR (100 MHz, CDCl3) $\delta 149.98,144.32,144.26,143.63,136.11,132.38,131.97$, $128.56,128.51,127.05,125.51,122.56,119.35,89.60,88.13,53.47,35.92,31.44,30.90$, $30.88,30.32,29.72,22.52,14.05,14.03$. (Figura 6)

Figura 6. Resonancia Magnética Nuclear 1H-RMN (400 MHz, CDCl3)

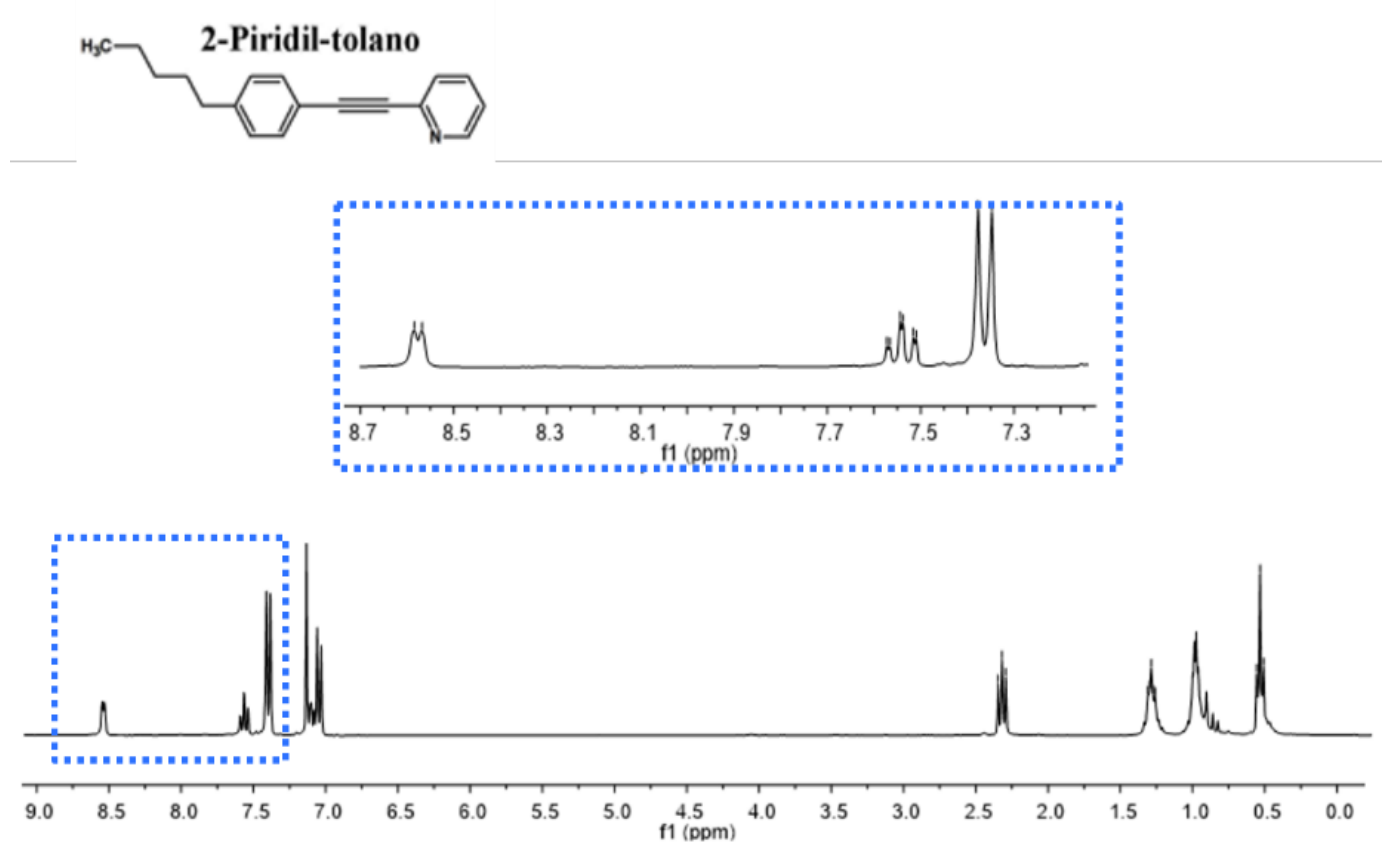

Fuente: Elaboración propia. 
Figura 7. Resonancia Magnética Nuclear 13C (400 MHz, CDCl3)

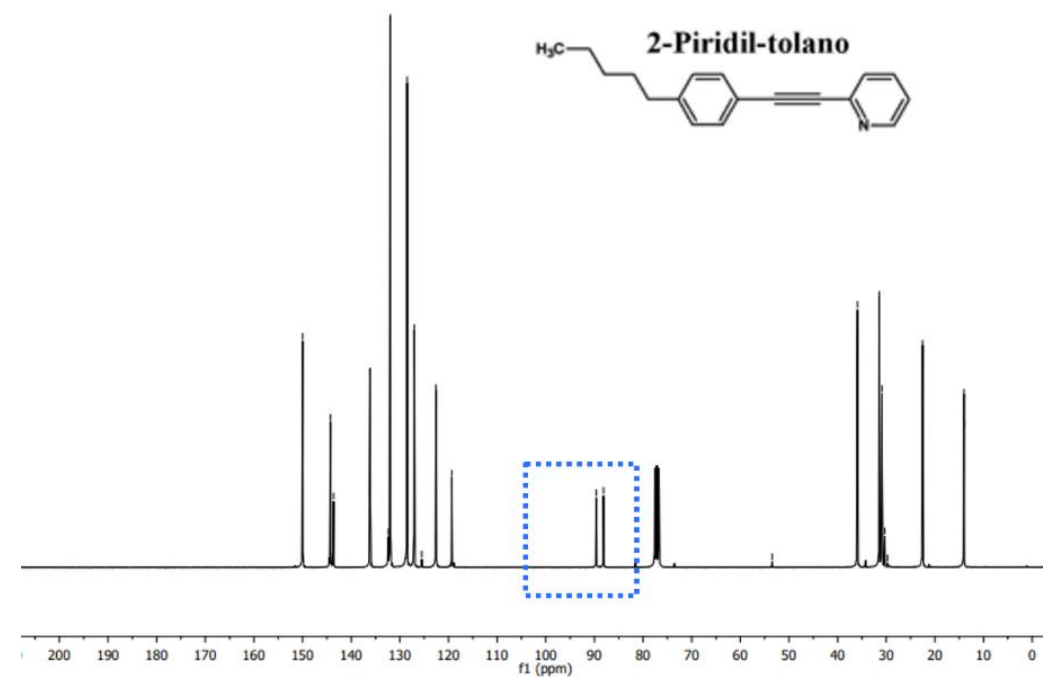

Fuente: Elaboración propia.

\section{Conclusiones:}

- En el presente trabajo mediante un acoplamiento de Sonogashira se sintetizaron el 2-piridil-tolano, 3-piridil-tolano y el 4-piridil-tolano a partir de 1-etinil-4pentilbenceno asistido por microondas en poco tiempo ( 2 horas), las tres estructuras fueron purificadas por Cromatografía de columna y caracterizadas por Resonancia Magnética Nuclear de Hidrógeno y Carbono, el tetracloruro de carbono interfiere con los enlaces de los compuestos polares y es tóxico, por lo que se utilizó un disolvente inerte marcado con deuterio específicamente cloroformo deuterado, puesto que el deuterio tiene diferente momento magnético que el hidrógeno, no da señal cuando se registra un espectro de RMN ajustado al protón. Se evidenció la formación de los Tolanos deseados, con un rendimiento de reacción del 71\% para 2-piridil-tolano, 73\% para 3-piridil-tolano y 78\% para 4-piridil-tolano. El uso de radiación por microondas es aprovechado adecuadamente para conseguir mejor selectividad en relación a los métodos convencionales, cuyos rendimientos no superan el 50\%, corroborando sus ventajas redactadas en bibliografía como ahorro energético, ahorro de disolventes y tiempos de reacción. A futuro se planea estudiarlos como posibles candidatos para la formación de cristales líquidos, se apuesta a la formación de enlaces de halógeno formando compuestos de hasta tres ligandos y compararlos con los enlaces de hidrógeno.

\section{Referencias bibliográficas:}

Bertus, P., Fecourt, F., Bauder, C., \& Pale, P. (2004). New Journal of Chemistry, 12.

Bunge, S., Krueger, K., Boyle , T., Rodríguez, M., Headly, T., \& Colvin, V. (2003). Materials Chemie, 1705-1709.

Cassar, L. (1975). Organomet. Chem, 253. 
Dick, H., \& Heck, F. (1975). Organomet. Chem, 259.

Dudley, G., Stiegman, A., \& Rosana, M. (2013). Correspondence on microwave effects in organic synthesis. Angewandte Chemie International Edition, 7918-7923.

Gabriel, C., Gabriel, S., Grant, E., Halstead, B., \& Mingos, D. (1998). Dielectric parameters relevant to microwave dielectric heating Chem. Soc. Chemical society reviews, 213-223.

Garro, R., Jiménez, P., \& Vega, J. (2013). Síntesis Asistida Por Microondas Del Poli ( Adipato de 1, 4 -Butadiol). . Rev. Iberoam. Polímeros, 304-312.

Gawande, M., Bonifacio, V., \& Luque, R. (2012). Benign by design: Catalyst-free inwater, on-water green chemical methodologies in organic synthesis. Chemical Society Reviews, 5522-5551.

Gedye, R., Smith, F., Westaway, K., Ali, H., Baldisera, L., Laberge, L., \& Rousell, J. (1986). The use of microwave-ovens for rapid organic-synthesis. Tetrahedron letters, 279-282.

Giguere, R., Bray, T., Duncan, S., \& Majetich, G. (1986). Application of commercial microwave-ovens to organic-synthesis. Tetrahedron letters, 4945-4948.

Gou, L., Chipara, M., \& Zaleski, J. (2007). Convenient, Rapid Synthesis of Ag Nanowires. Chemistry of Materials, 1755-1760.

Hopkins, C., \& Collar, N. (2004). Tetrahedron Letters, 8087-8090.

Kamali, H., Khodaverdi, E., \& Hadizadeh, F. (2018). Ring-Opening Polymerization of PLGA-PEG-PLGA Triblock Copolymer in Supercritical Carbon Dioxide. Supercrit. Fluids, 9-15.

Kappe, O. (2004). Controlled Microwave Heating in Modern Organic Synthesis. Angewandte Chemie International Edition, 6250-6284.

Kappe, O., Pieber, B., \& Dallinger, D. (2013). Microwave effects in organic synthesis: Myth or reality? . Angewandte Chemie International Edition, 1088-1094.

Mazo, P., Rios, L., \& Restrepo, G. (2011). Síntesis de Poli Ácido Láctico y Poli Ricinoleato Empleando Calentamiento Por Microondas y Su Utilización En La Producción de Termoplasticos de Poliuretano. Polímeros, 83-89.

Nguyen, H., Horton , P., Hursthouse, M., Legon, A., \& Bruce, D. (2004). Journal of the American Chemical Society, 16-17.

Paresh, C. (2010). Size and Shape Dependent Second Order Nonlinear Optical Properties of Nanomaterials and Their Application in Biological and Chemical Sensing. Chemical reviews, 5332-5365.

Polshettiwar, V., \& Varma, R. (2008). Microwave-Assisted Organic Synthesis and Transformations Using Benign Reaction Media. Accounts of chemical research, 629-639.

Sonogashira, K. (2003). Organomet. Chem, 46-49.

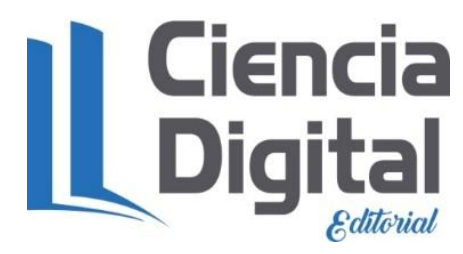




\section{PARA CITAR EL ARTÍCULO INDEXADO.}

Flores Fiallos, L. M., Villegas Freire, C. N., \& Guadalupe Alcoser, M. A. (2020). Síntesis de PIRIDIL-tolanos mediante un acoplamiento de sonogashira asistido por microondas. Ciencia Digital, 4(3), 95-106. https://doi.org/10.33262/cienciadigital.v4i3.1303

\section{¿Ciencia}

El artículo que se publica es de exclusiva responsabilidad de los autores y no necesariamente reflejan el pensamiento de la Revista Ciencia Digital.

El artículo queda en propiedad de la revista y, por tanto, su publicación parcial y/o total en otro medio tiene que ser autorizado por el director de la Revista Ciencia Digital.
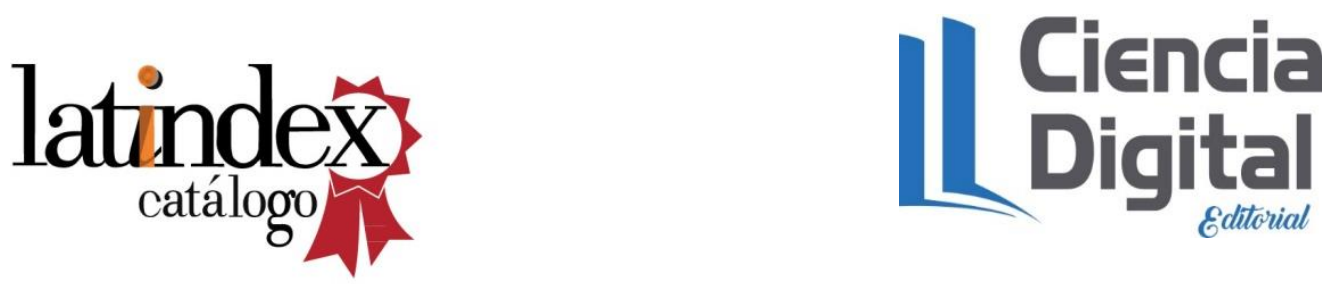